\title{
Flat-end tool orientation based on rotation-minimizing frame
}

\author{
Xiang Zou ${ }^{1} \cdot$ Hon-Yuen $\mathrm{Tam}^{2}$ (D) Hai-Yin $\mathrm{Xu}^{1} \cdot \mathrm{Ke} \mathrm{Shi}^{1}$
}

Received: 10 January 2019/Revised: 27 March 2019/Accepted: 8 July 2019/Published online: 15 August 2019

(C) The Author(s) 2019

\begin{abstract}
Well-designed tool orientation is crucial for the quality execution of five-axis machining with flat-end tools. Tool orientation can be specified in terms of tool inclination and tool tilt angles. For a given surface path, these aspects need to be specified for each cutter contact point along the path, because poor tool orientation choice can cause large axial acceleration of the machine tool, leading to inferior quality of the machined surface. The rotation-minimizing frame aims to reduce unnecessary frame rotation during movement. This concept has been adopted to develop tool orientation methods that allow variation in the inclination angle, the tilt angle, and both the inclination and tilt angles. The intention is to reduce unnecessary rotation of the tool frame as the tool follows a specified path. Evaluation was conducted in the context of five-axis flat-end tool machining. Based on these methods, tool orientation was planned along surface paths of a torus, sphere, and dome. Changes in tool orientation were always smooth. From the perspective of reducing tool orientation changes and axial acceleration, it was demonstrated that simultaneous variation of the inclination and tilt angles based on rotation minimization provided the best results.
\end{abstract}

Keywords Five-axis machining - Tool orientation · Rotation-minimizing frame $\cdot$ Numerical control

Hon-Yuen Tam

metamhy@cityu.edu.hk

1 School of Computer Science and Technology, Huazhong University of Science and Technology, Wuhan 430074, People's Republic of China

2 College of Science and Engineering, Department of Mechanical Engineering, City University of Hong Kong, Hong Kong, People's Republic of China

\section{Introduction}

Tool path planning for five-axis computer numerical control (CNC) machining has attracted significant interest in recent years. Constraints to the planning process arise from gouging avoidance, kinematic limits, singularity avoidance, cutting precision, and efficiency requirements. However, complete solutions to path planning have yet to be identified.

An attempt was made to divide the part surface into convex and non-convex regions, and to determine the optimal cutter orientation for local and rear gouging avoidance, by considering the offset of the machined surface and the offset cylinder of the cutter [1]. Maximization of the machining quality was achieved by identifying gouging-free tool paths, analyzing hyper-osculating circles that have third-order contact with the target surface, and formulating two-contact configurations as systems of algebraic constraints [2]. The concept of forbidden circles was proposed to avoid singularities in five-axis machining [3]. The problem was considered two-dimensional, as a three-dimensional "singular cone" associated with tool orientation was projected onto a two-dimensional space. Efforts were made to compensate for geometric errors to improve machining precision based on a generalized inverse kinematic model of the five-axis machine tool $[4,5]$. According to variations in cutting force and stability considerations along the tool path, efficient surface milling parameters were identified for tool path generation, the aim being to shorten the milling time [6].

Typical path planning involves generating an initial tool path, including the identification of cutter contact (CC) points and cutter positions, and construction of adjacent tool paths and $\mathrm{CC}$ points with consideration of factors such as material removal rate, scallop height, gouging, and 
machine dynamics. It is crucial to identify the tool orientation at each of the $\mathrm{CC}$ points. The focus of this paper is to address tool orientation for five-axis machining with flatend tools.

Tool orientation is an important factor that affects the surface quality of a machined part [7]. Existing research in tool orientation for five-axis machining has focused mainly on gouging avoidance, rotary axes movement optimization, and cutting efficiency.

To avoid local gouging, rear gouging, and global tool collision, search methods based on configuration space (C space) [8-10] were proposed to determine the tool orientation, in which the motion plan of the object was transformed into the motion plan of a point in the $\mathrm{C}$ space. Ezair and Elber [11] further utilized conservative computation of accessibility for a finite surface and orientation elements to ensure the correctness of collision-free orientation.

For chatter-free tool orientation, structural dynamics of the tool and work piece were analyzed in terms of cutter workpiece engagement coordinates [12]. An iterative search for gouging-free tool axis orientation was performed using the Nyquist criterion.

To maximize material removal and avoid local gouging, tool orientation was planned along the tool path to maintain a constant scallop height $[13,14]$. Decisions were made based on the local surface curvatures and analyses of the effective cutting radius $[15,16]$. In actuality, there is a trade-off between increasing material removal rate and reducing scallop height between adjacent paths [17].

Li et al. [18] presented a strategy for tool orientation planning, which divided a complex surface into regions based on both the Gaussian and mean curvatures, to reduce the rate of change in the tool orientation. Castagnetti et al. [19] proposed the concept of domain-of-admissible-toolorientation to minimize the movement of each rotary axis. Ho et al. [20] adopted a circular arc representation of tool path segments to smoothen tool orientation changes between adjacent CC points. Jun et al. [21] formalized a weight criterion in the $\mathrm{C}$ space, which consisted of the square root of variation of the tool tilt and inclination angles between two consecutive CC points, for smoothing the tool orientation.

$\mathrm{Hu}$ and Tang [22] focused on improving the dynamic performance of the machine tool and reducing the maximum angular acceleration at the rotary axes. An optimal work piece setup was first identified through a heuristic search. Subsequently, the tilt and yaw angles were found to optimize the tool orientation. An accurate cutter/work piece engagement model was developed by Zhang et al. [23] for optimizing the tool inclination and tilt angles for efficient and accurate machining. $\mathrm{Xu}$ [24] proposed an interpolation scheme for online calculation of tool orientation at each interpolated point to improve machining efficiency. Li et al. [25] suggested a two-stage process to reduce the cutter posture change between two adjacent cutter contact points. This was achieved by recognizing the range of cutter posture angles for collision avoidance and estimating the direction for smoothest posture change. Lin et al. [26] developed a method to change the tool orientation to avoid five-axis singularities and improve the texture of the machined surface.

The above literature review shows the following: (i) tool orientation can be represented by the tilt angle and inclination angle, (ii) tool orientation algorithms mainly involve finding the best angles based on certain objectives while satisfying certain requirements, and (iii) these algorithms should aim at stable and non-excessive rotary axes movement.

Farouki and $\mathrm{Li}$ [27] and Farouki et al. [28] used a rotation-minimizing frame (RMF) to reduce variation in tool orientation in five-axis machining with ball-end tools. The tool inclination angle was fixed to maintain constant cutting speed, and the component of the tool vector on the tangent plane was specified as the parallel transport vector. This resulted in smooth overall tool orientation variation, and the total tool rotation about the surface normal was smaller than that in Sturz's method. However, Han [29] pointed out that tool axis movement was not always small for an RMF with a parallel transport vector. Alternate RMFs for various applications were reviewed by Farouki [30]. It appears that methods favoring a small and stable tool axis speed improve efficiency and ensure robust machine tool performance.

In general five-axis machining with flat-end tools, both tool inclination and tilt angles may be varied during machining. Previous work has demonstrated the benefits of using an RMF to promote smooth changes in the tool axis. However, the cited works only considered changing the tool inclination angle, while the tilt angle remained constant. For cutting with ball-end tools and corner radius tools, a constant tilt angle implies a constant cutting speed. For cutting with flat-end tools, it may be advantageous to vary the tilt angle as well. This paper intends to address the use of an RMF to adjust the tool inclination angle and/or the tool tilt angle. Basic concepts pertaining to RMFs are reviewed in the next section. Section 3 presents methods for tool orientation for RMFs. Comparison of these methods, which includes tool paths on a torus, sphere, and dome, is conducted in Section 4. This is followed by a simulation of tool orientation changes on a free-form surface. The final section concludes the paper. 


\section{A review of RMFs}

Due to its superior rotation motion properties, RMFs have drawn much attention for diversified applications such as rigid body movement planning [31-35] and construction of RMFs on multi-curves and surfaces [36-40]. In this section, basic concepts related to RMFs are reviewed, and the elements of RMFs relevant to this study are introduced.

For an orthogonal frame $\boldsymbol{F}=\left(\boldsymbol{f}_{1}, \boldsymbol{f}_{2}, \boldsymbol{f}_{3}\right)$ moving along a curve $\boldsymbol{C}(\xi)$ in three-dimensional space, the movement is said to be rotation-minimizing with respect to a certain reference direction if the angular velocity of $\boldsymbol{F}$ has no component along that direction.

For convenience, when the reference direction is $f_{1}$, it is named the rotary vector, and $f_{2}$ and $f_{3}$ are the basic vectors. The angular velocity $\boldsymbol{\omega}$ of $\boldsymbol{F}$ can then be expressed as

$\omega=\boldsymbol{f}_{1} \times \boldsymbol{f}_{1}^{\prime} / \sigma$,

where $\sigma=\left|\boldsymbol{C}^{\prime}(\xi)\right|$ is the rate of change of arc length associated with the curve.

The derivative of the basic vectors with respect to $\xi$ become

$\left\{\begin{array}{l}\boldsymbol{f}_{2}^{\prime}=\sigma \omega \times \boldsymbol{f}_{2}=\left(\boldsymbol{f}_{1} \times \boldsymbol{f}_{1}^{\prime}\right) \times \boldsymbol{f}_{2}, \\ \boldsymbol{f}_{3}^{\prime}=\sigma \boldsymbol{\omega} \times \boldsymbol{f}_{3}=\left(\boldsymbol{f}_{1} \times \boldsymbol{f}_{1}^{\prime}\right) \times \boldsymbol{f}_{3} .\end{array}\right.$

Basic vectors $f_{2}$ and $f_{3}$ do not rotate instantaneously about $f_{1}$, and their derivatives must be parallel to $f_{1}$ as

$\left\{\begin{array}{l}\boldsymbol{f}_{2}^{\prime} \times \boldsymbol{f}_{1}=0 \\ \boldsymbol{f}_{3}^{\prime} \times \boldsymbol{f}_{1}=0 .\end{array}\right.$

For an orthogonal frame moving along a three-dimensional space curve, with one of the frame vectors (i.e., $f_{1}$ ) aligned to certain reference direction, the frame is still free to rotate about that reference direction. According to the concept behind the RMF, $f_{2}$ and $f_{3}$ are selected so that they do not rotate instantaneously about $f_{1}$. In other words, there is no "unnecessary rotation" of the frame about the rotary vector as the frame moves along the space curve.

\section{RMF-based tool orientation}

Figure 1 shows surface $\boldsymbol{S}(u, v)$ parameterized by $u$ and $v$, and tool path $\boldsymbol{C}(\xi)=\boldsymbol{S}(u(\xi), v(\xi))$ on the surface. The surface is smooth and $\frac{\partial S}{\partial u} \times \frac{\partial S}{\partial v}$ does not vanish on the surface. Let $\boldsymbol{n}$ be the unit surface normal, $\boldsymbol{t}$ the unit curve tangent that points to the feed direction, and $\boldsymbol{k}=\boldsymbol{t} \times \boldsymbol{n}$ the unit bi-normal at the CC point $\boldsymbol{P}$ on the path. From computational geometry, $\left[\begin{array}{l}\boldsymbol{t}^{\prime} \\ \boldsymbol{k}^{\prime} \\ \boldsymbol{n}^{\prime}\end{array}\right]=\sigma\left[\begin{array}{ccc}0 & -\kappa_{\mathrm{g}} & \kappa_{\mathrm{n}} \\ \kappa_{\mathrm{g}} & 0 & \tau_{\mathrm{g}} \\ -\kappa_{\mathrm{n}} & -\tau_{\mathrm{g}} & 0\end{array}\right]\left[\begin{array}{l}\boldsymbol{t} \\ \boldsymbol{k} \\ \boldsymbol{n}\end{array}\right]$,

where $\kappa_{\mathrm{g}}, \kappa_{\mathrm{n}}$, and $\tau_{\mathrm{g}}$ are the geodesic curvature, normal curvature, and geodesic torsion, respectively. Vector $\boldsymbol{T}$ is the unit tool vector. Inclination angle $\alpha$ is the angle between $\boldsymbol{n}$ and $\boldsymbol{T}$. Vector $\boldsymbol{m}$ is the unit vector aligned to the projection of $\boldsymbol{T}$ on the tangent plane at $P$. Tilt angle $\beta$ is the angle between $\boldsymbol{t}$ and $\boldsymbol{m}$. Vectors $\boldsymbol{T}$ and $\boldsymbol{m}$ can be written as

$\boldsymbol{T}=\cos \alpha \boldsymbol{n}+\sin \alpha \boldsymbol{m}$,

$\boldsymbol{m}=\cos \beta \boldsymbol{t}+\sin \beta \boldsymbol{k}$.

In five-axis machining, tool orientation must be prescribed as it follows path $\boldsymbol{C}$ to machine surface $\boldsymbol{S}$. Tool orientation can be treated as selecting $\alpha$ and $\beta$ along the path.

In this section, RMFs are constructed according to various settings of the inclination and tilt angles. These include the following: (i) varying the tilt angle and maintaining a fixed inclination angle, (ii) varying the inclination angle and maintaining a fixed tilt angle, and (iii) varying both the tilt and inclination angles.

\subsection{Varying the tilt angle}

In this setting, tilt angle $\beta$ is allowed to vary while inclination angle $\alpha$ is fixed. This can be considered as a direct application of Farouki's method [27]. As shown in Fig. 1, when $\alpha$ is fixed, the component of $\boldsymbol{T}$ along the normal direction is also fixed. When $\beta$ is allowed to vary, the component of $\boldsymbol{T}$ on the tangent plane may vary.

$\operatorname{RMF}(\boldsymbol{n}, \boldsymbol{h}, \boldsymbol{m})$ is selected with $\boldsymbol{n}$ as the rotary vector and $\boldsymbol{m}$ and $\boldsymbol{h}$ as basic vectors, where $\boldsymbol{h}=\boldsymbol{m} \times \boldsymbol{n}$. This choice of the RMF reduces variation in the basic vectors as rotary vector $\boldsymbol{n}$ varies along the tool path. For instance, the basic

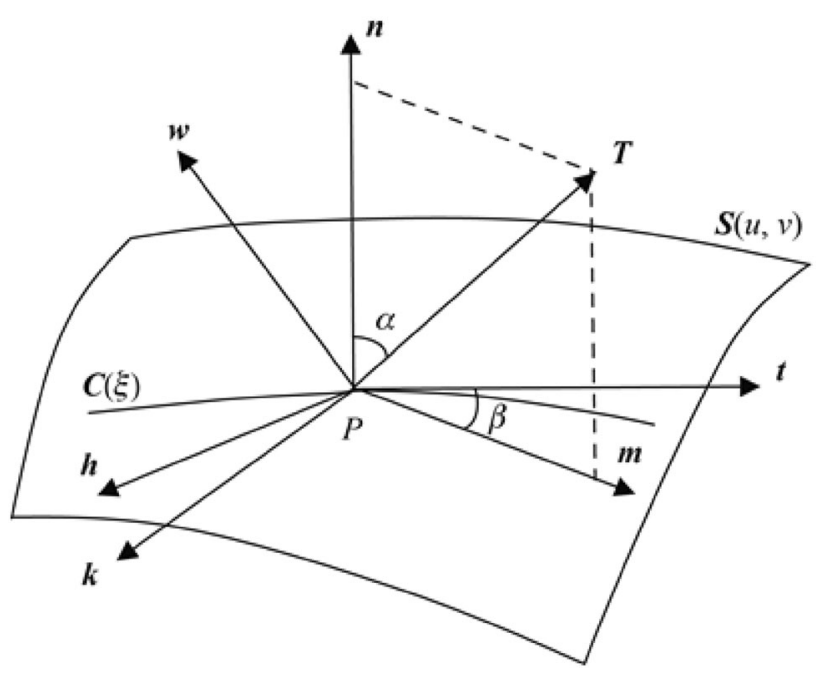

Fig. 1 Tool axis orientation along a tool path on a surface 
vectors do not rotate about $\boldsymbol{n}$ and their instantaneous change is parallel to $\boldsymbol{n}$. This rotation method is named RM I.

From Eqs. (4) and (6), the derivative of $\boldsymbol{m}$ with respect to $\xi$ along the curve can be written as

$\begin{aligned} \boldsymbol{m}^{\prime}= & \left(\beta^{\prime}-\sigma \kappa_{\mathrm{g}}\right)(-\sin \beta \boldsymbol{t}+\cos \beta \boldsymbol{k}) \\ & +\sigma\left(\kappa_{\mathrm{n}} \cos \beta+\tau_{\mathrm{g}} \sin \beta\right) \boldsymbol{n} .\end{aligned}$

As $\boldsymbol{t}$ and $\boldsymbol{k}$ are orthogonal to $\boldsymbol{n}$, and $\boldsymbol{m}^{\prime} \times \boldsymbol{n}=0$ according to Eq. (3), components of $\boldsymbol{m}^{\prime}$ along the directions of $\boldsymbol{t}$ and $\boldsymbol{k}$ in Eq. (7) must vanish. Obviously, $\sin \beta$ and $\cos \beta$ in these components cannot vanish simultaneously. Hence, the remaining option for satisfying $\boldsymbol{m}^{\prime} \times \boldsymbol{n}=0$ is

$\beta^{\prime}-\sigma \kappa_{\mathrm{g}}=0$,

and Eq. (7) can be simplified as

$\boldsymbol{m}^{\prime}=\sigma\left(\kappa_{\mathrm{n}} \cos \beta+\tau_{\mathrm{g}} \sin \beta\right) \boldsymbol{n}$.

Integrating Eq. (8) leads to

$\beta(\xi)=\beta_{0}+\int_{0}^{\xi} \sigma \kappa_{\mathrm{g}} \mathrm{d} \xi$,

where $\beta_{0}$ is an integration constant.

Computation of the tool orientation $\boldsymbol{T}$ requires selection of the fixed tool inclination angle $\alpha$ and the initial tilt angle $\beta_{0}$. Then, $\boldsymbol{T}$ can be calculated using Eqs. (5) and (6), and $\beta(\xi)$ can be evaluated numerically using Eq. (10) as the tool moves along the tool path.

Furthermore, from Eq. (5), the derivative of $\boldsymbol{T}$ is

$\boldsymbol{T}^{\prime}=\cos \alpha \boldsymbol{n}^{\prime}+\sin \alpha \boldsymbol{m}^{\prime}$,

which can be written as

$\boldsymbol{T}^{\prime}=\sigma\left(\cos \alpha\left(\kappa_{\mathrm{n}} \sin \beta-\tau_{\mathrm{g}} \cos \beta\right) \boldsymbol{h}+\left(\kappa_{\mathrm{n}} \cos \beta+\tau_{\mathrm{g}} \sin \beta\right) \boldsymbol{w}\right)$.

Using Eqs. (4) and (9) and rotation transformations, $\boldsymbol{t}=-\sin \beta \boldsymbol{h}+\cos \beta \boldsymbol{m}, \quad \boldsymbol{k}=\cos \beta \boldsymbol{h}+\sin \beta \boldsymbol{m}, \quad$ and $\boldsymbol{w}=\boldsymbol{h} \times \boldsymbol{T}=-\cos \alpha \boldsymbol{m}+\sin \alpha \boldsymbol{n}$.

\subsection{Varying the inclination angle}

In this setting, inclination angle $\alpha$ is allowed to vary while tilt angle $\beta$ is fixed. As shown in Fig. $1, \boldsymbol{h}$ is normal to the $\boldsymbol{m}-\boldsymbol{n}$ plane and $\boldsymbol{T}$ is fixed on that plane. If $\beta$ is fixed, so is $\boldsymbol{h}$. When $\alpha$ varies, the plane, together with $\boldsymbol{T}$, rotates about $\boldsymbol{h}$.

$\operatorname{RMF}(\boldsymbol{h}, \boldsymbol{T}, \boldsymbol{w})$ is selected with $\boldsymbol{h}$ as the rotary vector and $\boldsymbol{T}$ and $\boldsymbol{w}$ as the basic vectors, where $\boldsymbol{w}=\boldsymbol{h} \times \boldsymbol{T}$. This choice of the RMF reduces variations in the basic vectors as rotary vector $\boldsymbol{h}$ varies along the tool path. For instance, the basic vectors do not rotate about $\boldsymbol{h}$ and their instantaneous change is along the direction of $\boldsymbol{h}$ only. This rotation method is named RM II.

From Eqs. (4) and (6) and using rotation transformation, the derivatives of $\boldsymbol{n}$ and $\boldsymbol{m}$ can respectively be written as $\boldsymbol{n}^{\prime}=\sigma\left(\left(\kappa_{\mathrm{n}} \sin \beta-\tau_{\mathrm{g}} \cos \beta\right) \boldsymbol{h}-\left(\kappa_{\mathrm{n}} \cos \beta+\tau_{\mathrm{g}} \sin \beta\right) \boldsymbol{m}\right)$,

$\boldsymbol{m}^{\prime}=\sigma\left(-\kappa_{\mathrm{g}} \boldsymbol{h}+\left(\kappa_{\mathrm{n}} \cos \beta+\tau_{\mathrm{g}} \sin \beta\right) \boldsymbol{n}\right)$.

It is noted that the term $-\sigma \kappa_{\mathrm{g}} \boldsymbol{h}$ is present in Eq. (14) but not in Eq. (7). This is because $\beta$ is fixed in RM II but is allowed to vary as $\beta^{\prime}=\sigma \kappa_{\mathrm{g}}$ in RM I.

From Eq. (5), the derivative of $\boldsymbol{T}$ can firstly be written as

$\boldsymbol{T}^{\prime}=-\alpha^{\prime} \sin \alpha \boldsymbol{n}+\cos \alpha \boldsymbol{n}^{\prime}+\alpha^{\prime} \cos \alpha \boldsymbol{m}+\sin \alpha \boldsymbol{m}^{\prime}$,

and then rearranged using Eqs. (13) and (14) as

$\begin{aligned} \boldsymbol{T}^{\prime}= & \left(\alpha^{\prime}-\sigma\left(\kappa_{\mathrm{n}} \cos \beta+\tau_{\mathrm{g}} \sin \beta\right)\right)(\cos \alpha \boldsymbol{m}-\sin \alpha \boldsymbol{n}) \\ & +\sigma\left(\cos \alpha\left(\kappa_{\mathrm{n}} \sin \beta-\tau_{\mathrm{g}} \cos \beta\right)-\kappa_{\mathrm{g}} \sin \alpha\right) \boldsymbol{h} .\end{aligned}$

As $\boldsymbol{m}$ and $\boldsymbol{n}$ are orthogonal to $\boldsymbol{h}$, and $\boldsymbol{T}^{\prime} \times \boldsymbol{h}=0$ according to Eq. (3), the components of $\boldsymbol{T}^{\prime}$ along directions of $\boldsymbol{m}$ and $\boldsymbol{n}$ in Eq. (16) must vanish. Obviously, $\sin \alpha$ and $\cos \alpha$ in these components cannot vanish simultaneously. Hence, the remaining option for satisfying $\boldsymbol{T}^{\prime} \times \boldsymbol{h}=0$ is

$\alpha^{\prime}-\sigma\left(\kappa_{\mathrm{n}} \cos \beta+\tau_{\mathrm{g}} \sin \beta\right)=0$.

Equation (16) can then be simplified as

$\boldsymbol{T}^{\prime}=\sigma\left(\cos \alpha\left(\kappa_{\mathrm{n}} \sin \beta-\tau_{\mathrm{g}} \cos \beta\right)-\kappa_{\mathrm{g}} \sin \alpha\right) \boldsymbol{h}$.

Integrating Eq. (17) leads to

$\alpha(\xi)=\alpha_{0}+\int_{0}^{\xi} \sigma\left(\kappa_{\mathrm{n}} \cos \beta+\tau_{\mathrm{g}} \sin \beta\right) \mathrm{d} \xi$

where $\alpha_{0}$ is an integration constant.

Computation of tool orientation $\boldsymbol{T}$ requires selection of fixed tool tilt angle $\beta$ and initial inclination angle $\alpha_{0}$. Then, $\boldsymbol{T}$ can be calculated using Eqs. (5) and (6), and $\alpha(\xi)$ can be evaluated numerically using Eq. (19) as the tool moves along the path.

\subsection{Varying the tilt and inclination angles (dual RMF)}

To adapt to the local surface geometry more successfully, it may be preferable for the tool to be able to rotate about the normal direction, and to vary its inclination as it goes to the next tool contact point along the path. Thus, in a more general setting, this implies that both the inclination angle $\alpha$ and tilt angle $\beta$ are allowed to vary to allow more flexibility for orienting the tool.

Change in the tool orientation involves a two-sequential rotation of the tool axis. An RMF is employed so that 
variation of the basic vectors is reduced in each rotation by suppressing the rotation of the basic vectors about the rotary vector. Thus, this sequential rotation method is named dual RMF (DRM), which combines the primary RMF (PRMF) with the secondary RMF (SRMF).

In PRMF, the tool is set to rotate about the normal axis. Thus, $\boldsymbol{n}$ is selected as the rotary vector, with $\boldsymbol{h}$ and $\boldsymbol{m}$ as the basic vectors. Tilt angle $\beta$ is allowed to vary while inclination angle $\alpha$ is fixed. In other words, the PRMF is the same as RM I, and Eq. (8) is applicable to the determination of $\beta$.

In SRMF, the tool is set to rotate about $\boldsymbol{h}$, one of the basic vectors in PRMF. Thus, $\boldsymbol{h}$ is selected as the rotary vector, with $\boldsymbol{T}$ and $\boldsymbol{w}$ as the basic vectors. Inclination angle $\alpha$ is allowed to vary while tilt angle $\beta$ is fixed.

From Eq. (5), the derivative of $\boldsymbol{T}$ with respect to $\xi$ can be written as

$\boldsymbol{T}^{\prime}=-\alpha^{\prime} \sin \alpha \boldsymbol{n}+\cos \alpha \boldsymbol{n}^{\prime}+\alpha^{\prime} \cos \alpha \boldsymbol{m}+\sin \alpha \boldsymbol{m}^{\prime}$.

Here, $\boldsymbol{n}$ does not depend on $\beta$ but $\boldsymbol{m}$ does. The derivation of $\boldsymbol{n}^{\prime}$ in Eq. (13) does not involve any assumption with regard to $\beta$, and it can be used to express $\boldsymbol{n}^{\prime}$ in Eq. (20). Conversely, the derivation of $\boldsymbol{m}^{\prime}$ in Eq. (14) involves assumptions with regard to $\beta$ and it cannot be used. On the other hand, as the determination of $\beta$ in PRMF and that in RM I are exactly the same, Eq. (9) can be used to express $\boldsymbol{m}^{\prime}$. Therefore, Eq. (20) may be modified to

$$
\begin{aligned}
\boldsymbol{T}^{\prime}= & -\alpha^{\prime} \sin \alpha \boldsymbol{n}+\cos \alpha \cdot \sigma\left(\left(\kappa_{\mathrm{n}} \sin \beta-\tau_{\mathrm{g}} \cos \beta\right) \boldsymbol{h}\right. \\
& \left.-\left(\kappa_{\mathrm{n}} \cos \beta+\tau_{\mathrm{g}} \sin \beta\right) \boldsymbol{m}\right) \\
& +\alpha^{\prime} \cos \alpha \boldsymbol{m}+\sin \alpha \cdot \sigma\left(\kappa_{\mathrm{n}} \cos \beta+\tau_{\mathrm{g}} \sin \beta\right) \boldsymbol{n} \\
= & \left(\alpha^{\prime}-\sigma\left(\kappa_{n} \cos \beta+\tau_{\mathrm{g}} \sin \beta\right)\right)(\cos \alpha \boldsymbol{m}-\sin \alpha \boldsymbol{n}) \\
& +\sigma \cos \alpha\left(\kappa_{\mathrm{n}} \sin \beta-\tau_{\mathrm{g}} \cos \beta\right) \boldsymbol{h} .
\end{aligned}
$$

As the components of $\boldsymbol{T}^{\prime}$ along the directions of $\boldsymbol{m}$ and $\boldsymbol{n}$ in Eq. (21) must vanish, the same restriction on $\boldsymbol{T}^{\prime}$ needs to be imposed here (as in Eq. (17)), and $\boldsymbol{T}^{\prime}$ can then be further simplified as

$\boldsymbol{T}^{\prime}=\sigma \cos \alpha\left(\kappa_{\mathrm{n}} \sin \beta-\tau_{\mathrm{g}} \cos \beta\right) \boldsymbol{h}$.

Computation of tool orientation $\boldsymbol{T}$ requires selection of initial inclination angle $\alpha_{0}$ and initial tilt angle $\beta_{0}$. Then, $\alpha(\xi)$ and $\beta(\xi)$ can be evaluated numerically using Eqs. (19) and (10) respectively along the tool path, and $\boldsymbol{T}$ can be calculated using Eqs. (5) and (6) (see Fig. 2).

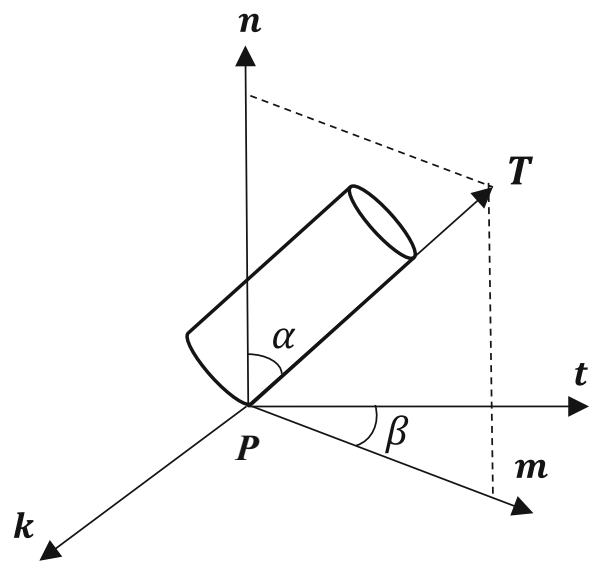

Fig. 2 Five-axis milling with flat-end tool

\section{Comparison of methods}

\subsection{Preparation}

\subsubsection{Kinematics}

Tool contact location and tool orientation information (together with tool and cutting parameters) are translated to axis command for the execution of five-axis machining [26]. The translation is conducted according to the kinematic chain of a machine. There are several common kinematic chains in five-axis machine tools [23-25]. For an $A-B$ type five-axis machine (i.e., an orientable-spindle machine), rotary axes $A$ and $B$ are parallel to the translation axes $X$ and $Y$.

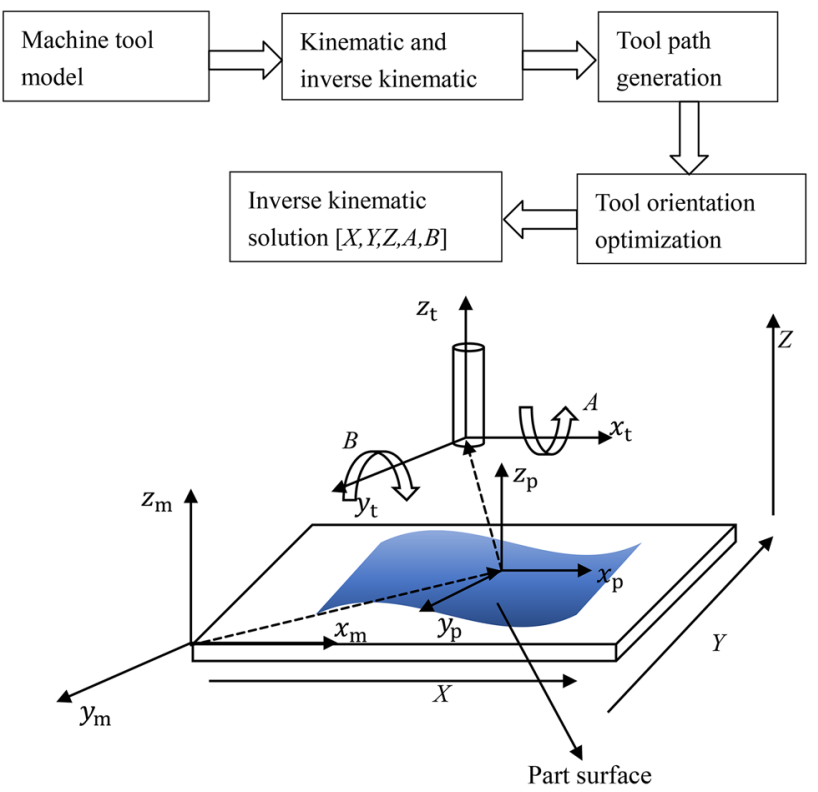

Fig. 3 Five-axis milling with flat-end tool 
Such a machine model (see Fig. 3) is adopted in this paper for kinematics calculations. The tool axis is along the $Z$-axis. $\left[x_{\mathrm{t}}, y_{\mathrm{t}}, z_{\mathrm{t}}\right],\left[x_{\mathrm{m}}, y_{\mathrm{m}}, z_{\mathrm{m}}\right]$ and $\left[x_{\mathrm{p}}, y_{\mathrm{p}}, z_{\mathrm{p}}\right]$ represent the tool coordinate system, machine coordinate system (MCS), and part coordinate system (PCS), respectively. Rotary axes $A$ and $B$ are parallel to translation axes $x_{\mathrm{t}}$ and $y_{\mathrm{t}}$. The kinematic equation can be established in MCS as

$\left[\begin{array}{ll}\boldsymbol{L} & \boldsymbol{T}\end{array}\right]=\boldsymbol{T}_{\text {rans,PCS } \rightarrow \mathrm{MCS}} \times \boldsymbol{T}_{\text {rans }}(X, Y, Z) \times \boldsymbol{T}_{\text {rans, }, \mathrm{TCS} \rightarrow \mathrm{PCS}}$

$$
\times \boldsymbol{R}_{\mathrm{ot}}(Y, B) \times \boldsymbol{R}_{\mathrm{ot}}(X, A) \times\left[\begin{array}{cc}
x_{o} & 0 \\
y_{0} & 0 \\
z_{0} & 1 \\
1 & 0
\end{array}\right],
$$

where $\boldsymbol{T}_{\text {rans }}$ represents the transport matrix and $\boldsymbol{R}_{\mathrm{ot}}$ is the rotation matrix; $\left(x_{0}, y_{0}, z_{0}\right)$ is the initial tool contact location, and $\left[\begin{array}{ll}\boldsymbol{L} & \boldsymbol{T}\end{array}\right]$ denotes the tool contact location and orientation in MCS. From Eq. (23), the relationship between the tool axis vector and rotary angles can be obtained as

$\boldsymbol{T}=\left[\begin{array}{c}T_{x} \\ T_{y} \\ T_{z}\end{array}\right]=\left[\begin{array}{c}\sin B \cos A \\ -\sin A \\ \cos B \cos A\end{array}\right]$.

Then, the rotary angles can be solved as

$A=\arcsin \left(-T_{y}\right), \quad B=\arctan \frac{T_{x}}{T_{z}}$.

In practical machining, $X, Y, Z, A$, and $B$ are generated after tool path generation and tool orientation determination. The focus of the current work is tool orientation determination with an RMF. After the tool path on the part surface has been generated, the tool orientation is determined (tilt and inclination angles). Then, rotary angles $A$ and $B$ can be calculated using Eq. (25). It should be mentioned that a singularity may arise during the calculation of $B$. Singularity avoidance is an important issue, but is not considered in the current work.

\subsubsection{Constraints on inclination and tilt angles}

For five-axis machine with a flat-end tool (see Fig. 2), the effective cutter radius $r_{\text {eff }}$ is expressed as

$r_{\mathrm{eff}}=a^{2} r^{2}\left(\frac{1+\tan ^{2} \theta}{a^{2}+r^{2} \tan ^{2} \theta}\right)$,

where $r$ is the tool radius, $a=r \sin \alpha \cos \beta$, and $\theta=\arctan (\tan \alpha \tan \beta)$. The effective cutter radius must not be larger than the radius of curvature at a CC point. In general, $\alpha$ should not be large enough to promote material removal.

Figure 4 shows an undesirable situation of rear gouging in which the rear of the tool interferes with the part surface.

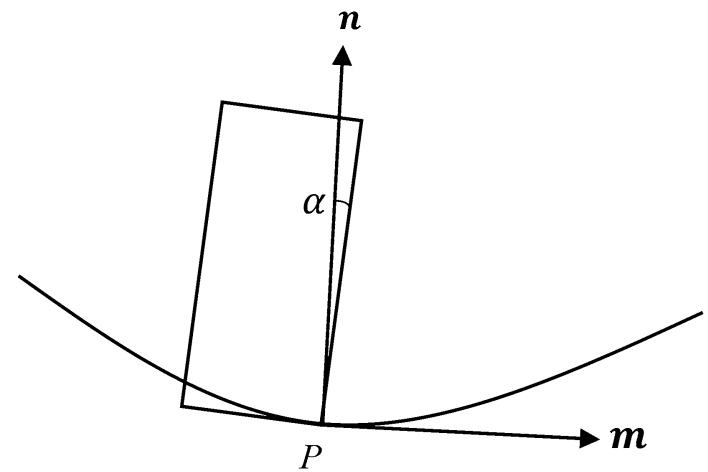

Fig. 4 Rear gouging

This can happen when $\alpha$ is too small as the tool passes a concave part of a surface. Thus, to avoid rear gouging, $\alpha$ should not be too small. Further, $\beta$ should be small so that $\theta$ can be small, allowing $r_{\text {eff }}$ to be large.

These considerations should be accounted for in the selection of the initial inclination and tilt angles. Other issues, such as collision avoidance and material removal efficiency, are also important for tool path and orientation planning; however, these aspects are beyond the scope of this paper.

\subsubsection{Evaluation}

The focus of the evaluation is to reduce the variation of the tool orientation. Small variation is preferred as it implies that the orientation of the tool need not change rapidly along a tool path, and less demand for rapid response is exerted on the rotary axes of the machine.

Variation in the tool orientation can be evaluated using the rate of change of the unit tool vector $\left|\boldsymbol{T}^{\prime}\right|$, which can be readily computed using Eqs. (12), (18), and (22) for the three methods. A small $\left|\boldsymbol{T}^{\prime}\right|$ suggests stable rotational movement of the rotary axes.

Changes in the rotary variables present another point for evaluation. Rotary angles $A$ and $B$ can be computed along the path using the kinematic model in Eq. (23). For three consecutive path points, values of the corresponding rotary angles $\left(A_{i-1}, B_{i-1}\right),\left(A_{i}, B_{i}\right)$, and $\left(A_{i+1}, B_{i+1}\right)$ can be obtained and acceleration terms

$$
\left\{\begin{array}{l}
S_{\mathrm{RA}}=\frac{\frac{A_{i+1}-A_{i}}{L_{i+1}}-\frac{A_{i}-A_{i-1}}{L_{i}}}{L_{i+1}+L_{i}}, \\
S_{\mathrm{RB}}=\frac{\frac{B_{i+1}-B_{i}}{L_{i+1}}-\frac{B_{i}-B_{i-1}}{L_{i}}}{L_{i+1}+L_{i}}, \\
S_{\mathrm{RAB}}=\sqrt{S_{\mathrm{RA}}^{2}+S_{\mathrm{RB}}^{2}},
\end{array}\right.
$$


can be computed to reflect demands on the rotary axes for changes in the angular velocity. Here, $L_{i}$ and $L_{i+1}$ correspond to the arc lengths between these path points.

\subsection{Comparison results}

Three sets of comparisons are reported. To facilitate comparison, the first and second sets are based on the paths used by Farouki and $\mathrm{Li}$ [27]. Tool orientation variation and rotary axes movement are computed along paths on these surfaces. Results for RM I, RM II, and DRM are indexed as 1, 2, and 3, respectively, in Figs. 5-17.

\subsubsection{Torus}

The torus surface in Cartesian space is defined as $\boldsymbol{S}(u, v)=((R+d \cos v) \cos u,(R+d \cos v) \sin u, d \sin v)$,

with $R=200 \mathrm{~mm}, d=100 \mathrm{~mm}$, and $u, v \in[0,2 \pi]$, and the tool path to be used is defined in the $(u, v)$ parametric space as

$\boldsymbol{C}(\xi)=(u(\xi), v(\xi))=\left(\frac{1}{2} \pi \xi, \frac{1}{2} \pi \xi\right)$,

with $\xi \in[0,1]$. Grid size $\Delta \xi=0.01$ is used in the experiments. Tool vector $\boldsymbol{T}$ along the path is depicted in Fig. 5 for the three methods. Initial angles $\alpha_{0}=\beta_{0}=5^{\circ}$ are found to give reasonable tool orientation and are adopted.

For RM I with $\alpha$ fixed at $5^{\circ}$, the tool vector is close to the surface normal $\boldsymbol{n}$. Notably, the actual tool location also depends on the inclination angle $\beta$, which varies along the path. Thus, the tool vector points almost horizontally at the start. Subsequently, the tool vector gradually changes together with the surface normal, and points almost vertically upwards near the end.

For RM II with $\beta$ fixed at $5^{\circ}$, the tool vector simply stays close to the $\boldsymbol{t}$-n plane. Initially, it also points almost

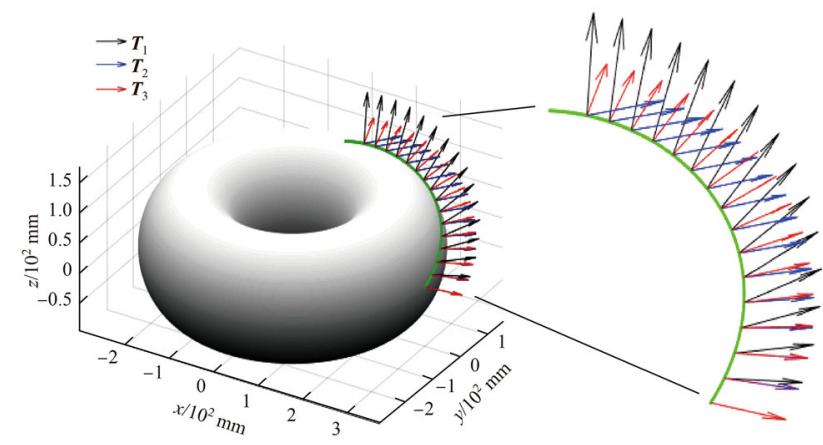

Fig. 5 Tool orientation on torus RM I $\left(\boldsymbol{T}_{1}\right)$, RM II $\left(\boldsymbol{T}_{2}\right)$, DRM $\left(\boldsymbol{T}_{3}\right)$

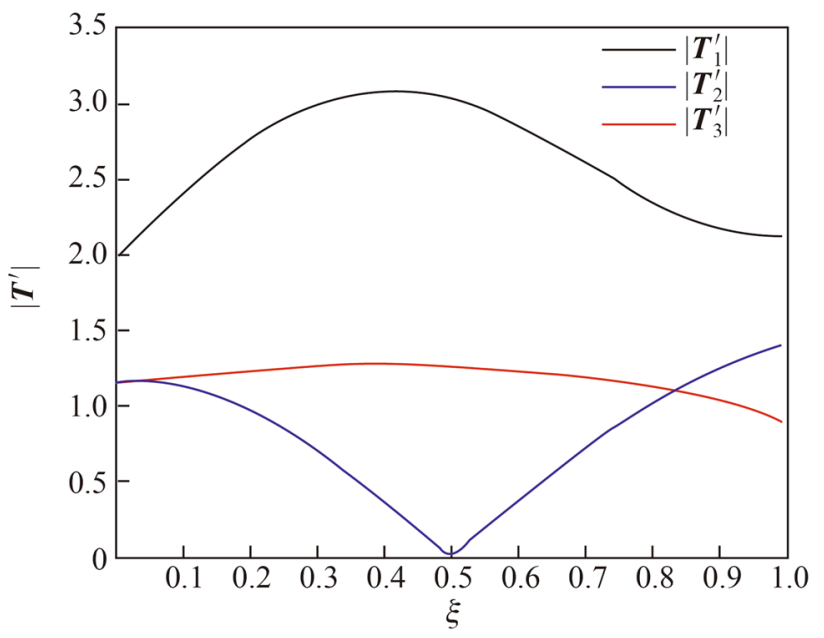

Fig. 6 Rate of tool orientation changes $\left|\boldsymbol{T}^{\prime}\right|$ on torus RM I $\left(\left|\boldsymbol{T}_{1}^{\prime}\right|\right)$, RM II $\left(\left|\boldsymbol{T}_{2}^{\prime}\right|\right)$, DRM $\left(\left|\boldsymbol{T}_{3}^{\prime}\right|\right)$

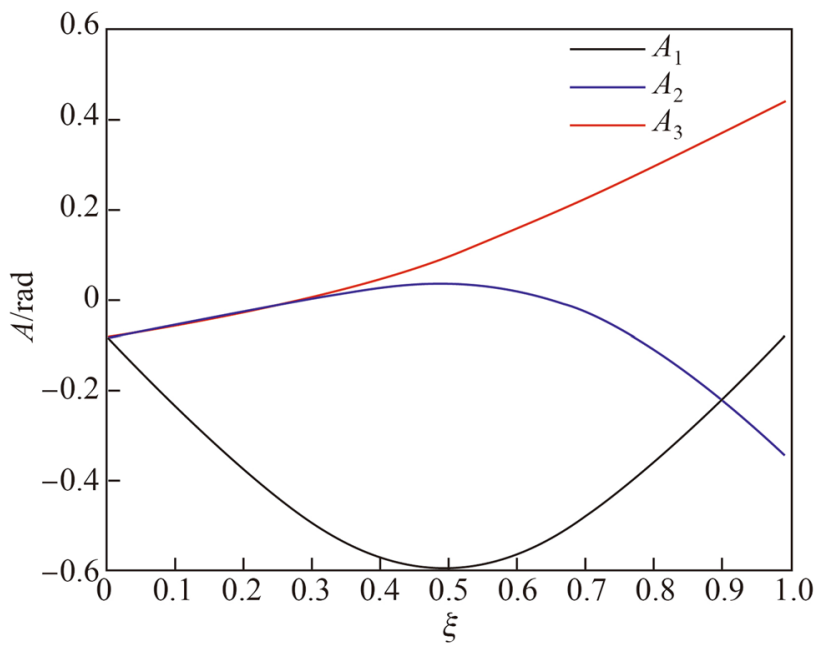

Fig. 7 Rotary angle $A$ along path on torus RM I $\left(A_{1}\right)$, RM II $\left(A_{2}\right)$, $\operatorname{DRM}\left(A_{3}\right)$

horizontally, then it gradually tilts away from $\boldsymbol{n}$ as the tool moves along the path.

For DRM, the tool vector falls on the $\boldsymbol{m}$ - $\boldsymbol{n}$ plane. It has the same initial orientation as in RM I and RM II. Then, it progressively inclines away from the $\boldsymbol{t}$ - $\boldsymbol{n}$ plane, and also tilts away from $\boldsymbol{n}$ along the path.

A comparison of the rate of change of tool orientation $\left|\boldsymbol{T}^{\prime}\right|$ for the three methods is given in Fig. 6. For RM I, $\left|\boldsymbol{T}^{\prime}\right|$ starts near 2 and then goes above 3 before coming down. For RM II, $\left|\boldsymbol{T}^{\prime}\right|$ starts near 1.2 and decreases to zero, and then goes up to about 1.4. It appears that the tool tilt direction reverses, leading to the intermediate zero $\left|\boldsymbol{T}^{\prime}\right| .\left|\boldsymbol{T}^{\prime}\right|$ also starts near 1.2 for DRM. However, its trend appears rather steady and $\left|\boldsymbol{T}^{\prime}\right|$ stays close to 1 throughout the path. Thus, RM I has the largest $\left|\boldsymbol{T}^{\prime}\right|$. The average $\left|\boldsymbol{T}^{\prime}\right|$ values are 


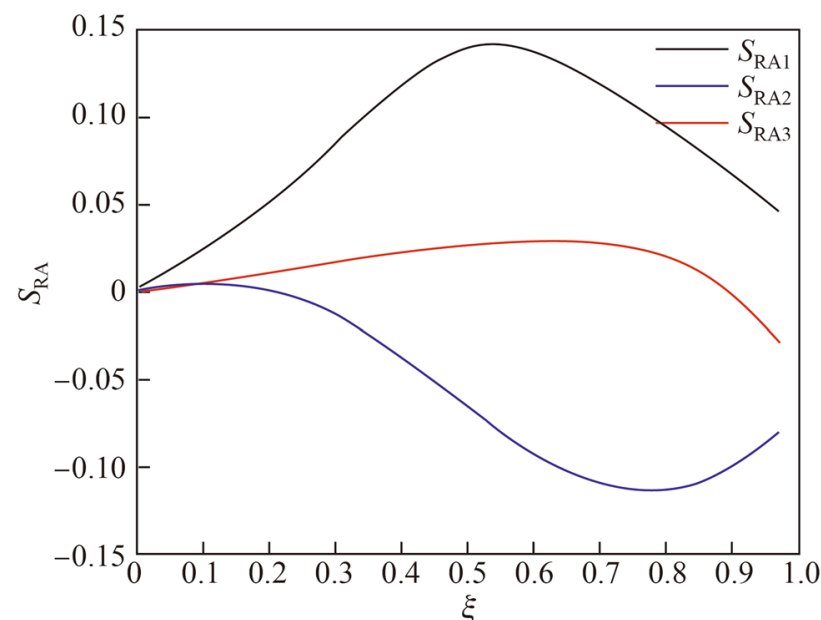

Fig. $8 S_{\mathrm{RA}}$ along path on torus RM I $\left(S_{\mathrm{RA} 1}\right)$, RM II $\left(S_{\mathrm{RA} 2}\right)$, DRM $\left(S_{\mathrm{RA} 3}\right)$

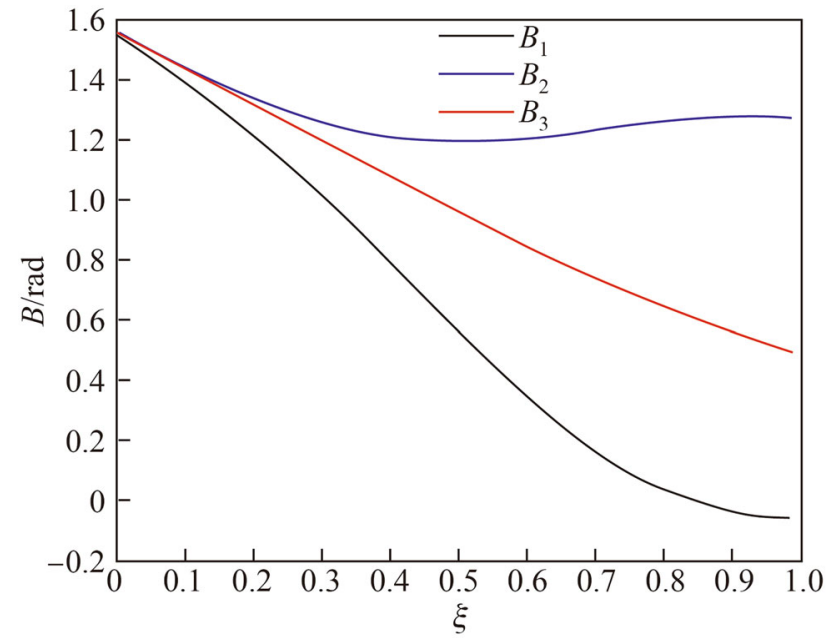

Fig. 9 Rotary angle $B$ along path on torus RM I $\left(B_{1}\right)$, RM II $\left(B_{2}\right)$, $\operatorname{DRM}\left(B_{3}\right)$

similar for RM II and DRM, but DRM has more steady $\left|\boldsymbol{T}^{\prime}\right|$ values and a smaller peak value than RM II.

Analytical representations of $\left|\boldsymbol{T}^{\prime}\right|$ for the three methods are given in Eqs. (12), (18), and (22). According to Eq. (22), $\left|\boldsymbol{T}^{\prime}\right|$ for DRM contains the term $\sigma \cos \alpha\left(\kappa_{\mathrm{n}} \sin \beta-\tau_{\mathrm{g}} \cos \beta\right) \boldsymbol{h}$ only. Thus, the change direction is orthogonal to $\boldsymbol{T}$. As $\alpha$ is typically small, the rate of change depends mainly on $\tau_{\mathrm{g}}$ if $\beta$ is small, and it also depends on $\kappa_{\mathrm{n}}$ if $\beta$ is not.

Besides the above term, Eq. (12) for RM I contains the term $\sigma\left(\kappa_{\mathrm{n}} \cos \beta+\tau_{\mathrm{g}} \sin \beta\right) \boldsymbol{w}$, which is orthogonal to that term. This suggests that RM I uses a larger $\left|\boldsymbol{T}^{\prime}\right|$ than DRM. The change direction is also orthogonal to $\boldsymbol{T}$ as $\boldsymbol{w}$ is orthogonal to $\boldsymbol{T}$.

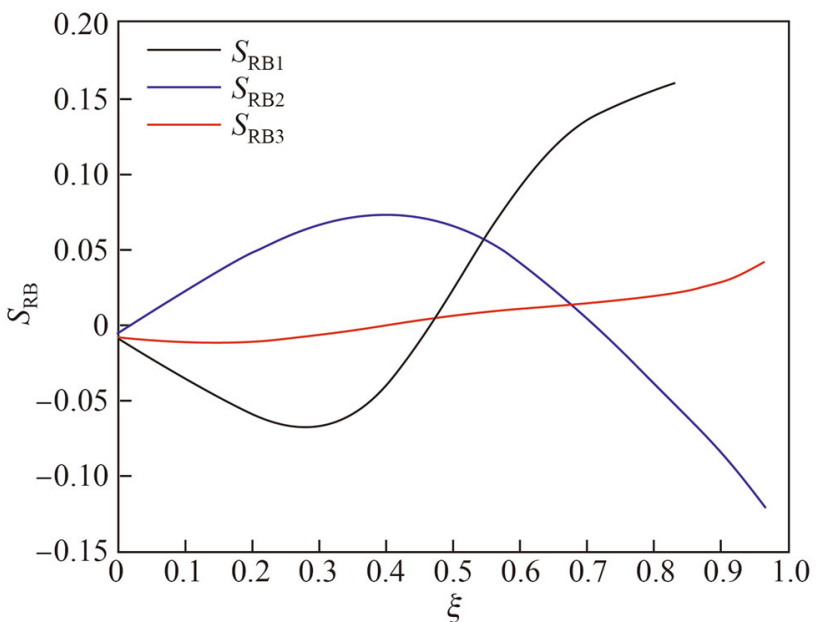

Fig. $10 S_{\mathrm{RB}}$ along path on torus RM I $\left(S_{\mathrm{RB} 1}\right)$, RM II $\left(S_{\mathrm{RB} 2}\right)$, DRM $\left(S_{\mathrm{RB} 3}\right)$

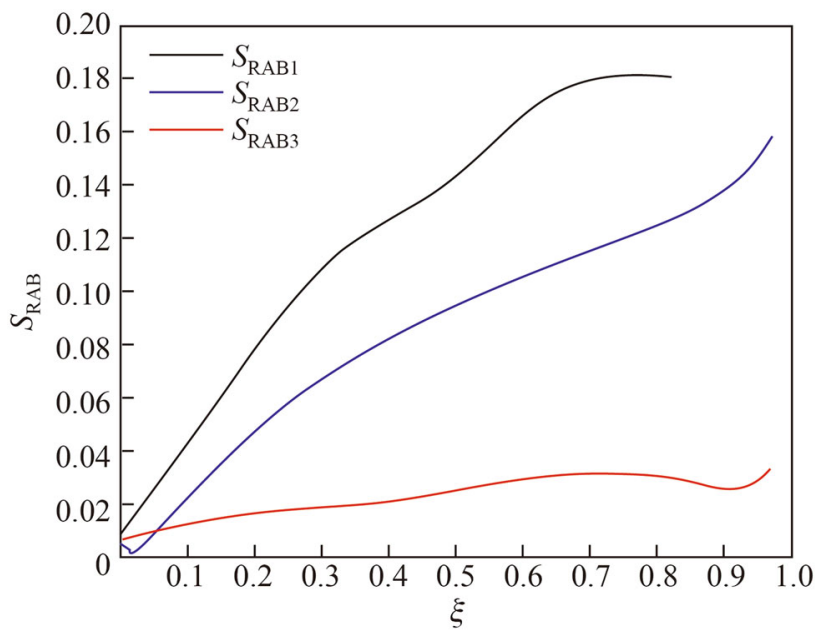

Fig. $11 S_{\mathrm{RAB}}$ along path on torus RM I $\left(S_{\mathrm{RAB} 1}\right)$, RM II $\left(S_{\mathrm{RAB} 2}\right)$, $\operatorname{DRM}\left(S_{\text {RAB3 }}\right)$

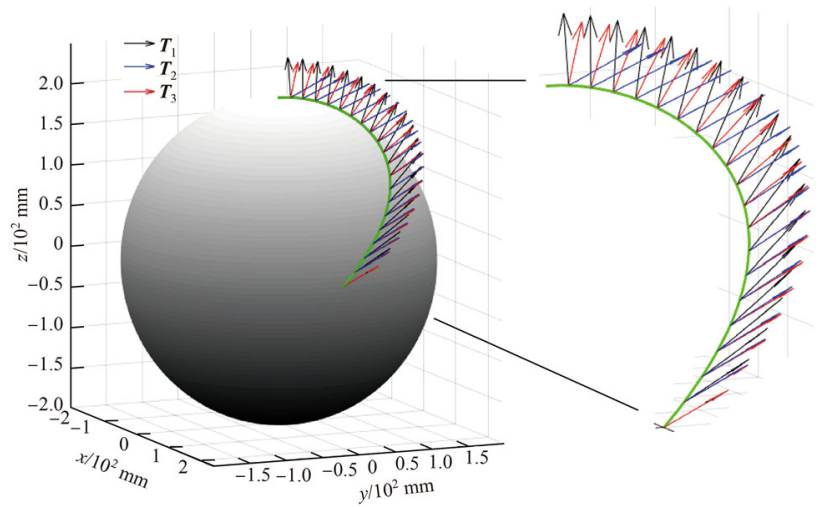

Fig. 12 Tool orientation on sphere RM I $\left(\boldsymbol{T}_{1}\right)$, RM II $\left(\boldsymbol{T}_{2}\right)$, DRM $\left(\boldsymbol{T}_{3}\right)$ 


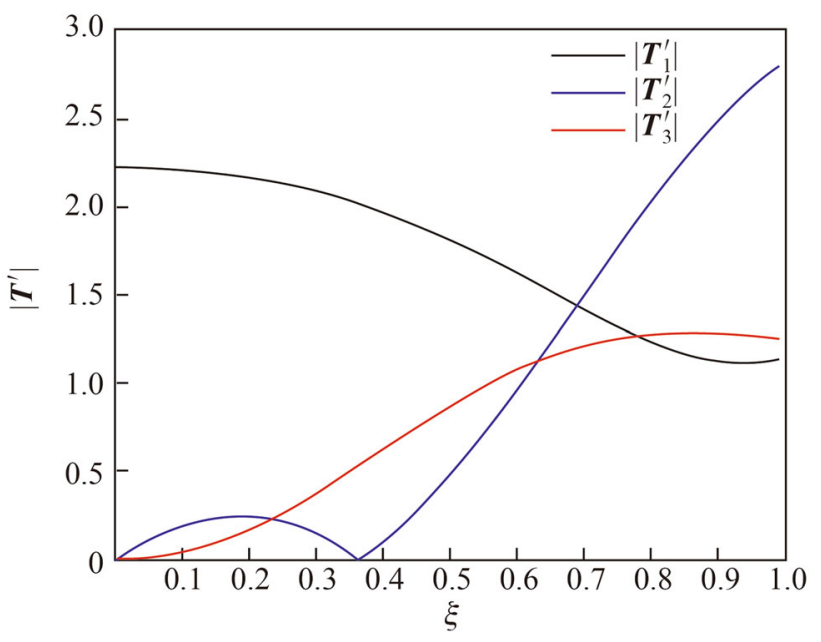

Fig. 13 Rate of tool orientation changes $\left|\boldsymbol{T}^{\prime}\right|$ on sphere RM I $\left(\left|\boldsymbol{T}_{1}^{\prime}\right|\right)$, RM II ( $\left.\left|\boldsymbol{T}_{2}^{\prime}\right|\right)$, DRM $\left(\left|\boldsymbol{T}_{3}^{\prime}\right|\right)$

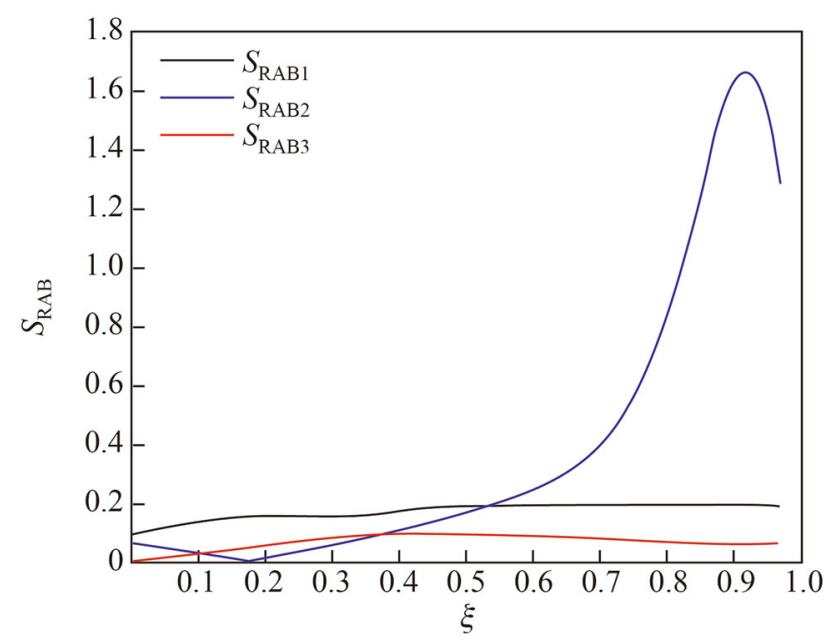

Fig. $14 S_{\mathrm{RAB}}$ along path on sphere RM I $\left(S_{\mathrm{RAB} 1}\right)$, RM II $\left(S_{\mathrm{RAB} 2}\right)$, $\operatorname{DRM}\left(S_{\mathrm{RAB} 3}\right)$

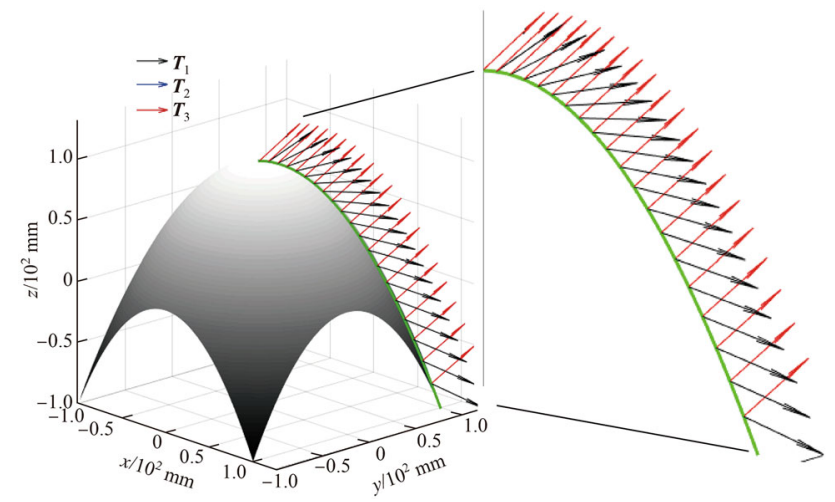

Fig. 15 Tool orientation on dome RM I $\left(\boldsymbol{T}_{1}\right)$, RM II $\left(\boldsymbol{T}_{2}\right)$, DRM $\left(\boldsymbol{T}_{3}\right)$

For RM II, the extra term in Eq. (18) is $-\sigma \kappa_{\mathrm{g}} \sin \alpha \boldsymbol{h}$. This indicates that the change direction is the same as that

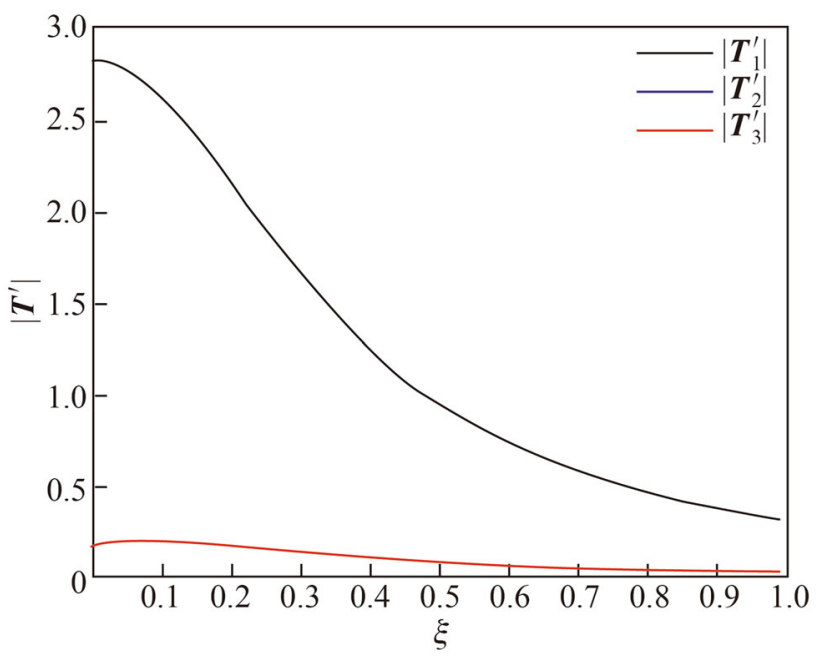

Fig. 16 Rate of tool orientation changes $\left|\boldsymbol{T}^{\prime}\right|$ on dome RM I $\left(\left|\boldsymbol{T}_{1}^{\prime}\right|\right)$, RM II (| $\left.\boldsymbol{T}_{2}^{\prime} \mid\right)$, DRM $\left(\left|\boldsymbol{T}_{3}^{\prime}\right|\right)$

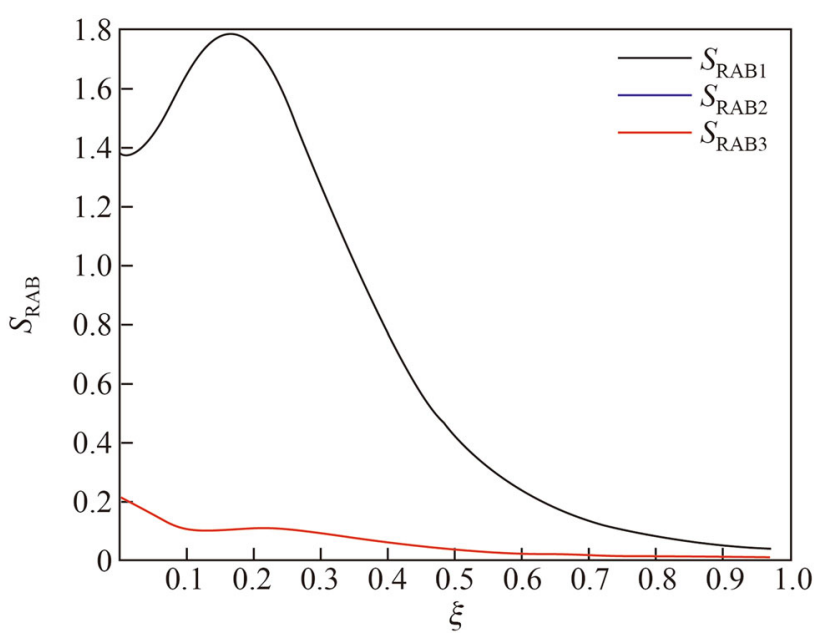

Fig. $17 S_{\mathrm{RAB}}$ along path on dome RM I $\left(S_{\mathrm{RAB} 1}\right)$, RM II $\left(S_{\mathrm{RAB} 2}\right)$, $\operatorname{DRM}\left(S_{\text {RAB3 }}\right)$

for DRM. While $\left|\boldsymbol{T}^{\prime}\right|$ depends on $\kappa_{\mathrm{n}}$ and $\tau_{\mathrm{g}}$ in DRM, it also depends on $\kappa_{\mathrm{g}}$ in RM II.

Rotary angles $A$ and $B$ and the corresponding acceleration terms $S_{\mathrm{RA}}$ and $S_{\mathrm{RB}}$ are shown in Figs. 7-10. The rotary angles start in the same manner for all the three methods because the tool orientation is the same initially (see Fig. 5). Subsequently, the angles assume quite different values as different methods are used to change the inclination and tilt angles. The acceleration terms also vary in different ways for the three methods (see Figs. 8 and 10). However, it is observed that DRM uses smaller acceleration than the other methods. This may be attributed to the facts that both the inclination and tilt angles are allowed to vary in DRM and that rotation minimization is adopted for 
those changes. The term $S_{\text {RAB }}$ is explained in Fig. 11. This term reflects the combined acceleration of the individual rotary axes and also indicates that DRM uses the smallest acceleration. It can be seen that $S_{\mathrm{RAB}}$ is the smallest for DRM, and $S_{\mathrm{RAB}}$ values for RM II are slightly smaller than those for RM I.

\subsubsection{Sphere}

The sphere surface is defined as

$S(u, v)=[R \cos u, R \cos v \sin u, R \sin v]$,

with $R=200 \mathrm{~mm}, u \in[0,2 \pi]$, and $v \in\left[-\frac{1}{2} \pi, \frac{1}{2} \pi\right]$. Tool path $\boldsymbol{C}(\xi)$ is as defined in Eq. (29). The same grid size is adopted, and initial $\alpha_{0}=45^{\circ}$ and $\beta_{0}=0^{\circ}$. For the three methods, the tool vector along the path is given in Fig. 12. Again, although the initial tool orientation is the same (i.e., aligned to the surface normal), subsequent tool orientation is quite different because the inclination and tilt angles are changed using different ways in the three methods.

The rate of change of tool orientation $\left|\boldsymbol{T}^{\prime}\right|$ is given in Fig. 13. The value of $\left|\boldsymbol{T}^{\prime}\right|$ for RM I is large in the beginning and drops to a value close to that of DRM in the end. Conversely, the value of $\left|\boldsymbol{T}^{\prime}\right|$ for RM II starts small but grows rapidly from mid-course onward. It seems that DRM has the smallest overall $\left|\boldsymbol{T}^{\prime}\right|$ value. Moreover, $\left|\boldsymbol{T}^{\prime}\right|$ is always smooth for DRM.

Figure 14 clearly shows that DRM has a relatively steady axial acceleration and the smallest $S_{\mathrm{RAB}}$. Both RM I and RM II use similar acceleration all the way until about two-thirds of the path. Then, a surge in acceleration occurs for RM II so as to keep up with the planned tool orientation. This may be attributed to the local geometry, in combination with the configuration of the machine tool, which might not favor orienting the tool with a fixed tilt angle in that vicinity. The surge is not obvious from the tool orientation (see Fig. 12) and its rate of change (see Fig. 13). The value of $S_{\text {RAB }}$ is small for both DRM and RM I. However, that of $\left|\boldsymbol{T}^{\prime}\right|$ is much larger for RM I.

\subsubsection{Dome}

The surface is defined as

$S(u, v)=\left[R u, R v, R\left(1-u^{2}-v^{2}\right)\right]$,

with $R=100 \mathrm{~mm}$ and $u, v \in[-1,1]$. The tool path is $\boldsymbol{C}(\xi)=(\xi, \xi)$ with $\xi \in[0,1]$. Initial $\alpha_{0}$ and $\beta_{0}$ are $45^{\circ}$ and $5^{\circ}$, respectively. The tool path and orientation are given in Fig. 15. The path starts at the top of the dome and ends at one of the base corners. For RM I, the tool axis maintains an angle of $45^{\circ}$ to the surface normal along the path. RM II allows changes in the inclination angle. The tool axis gradually approaches the normal direction as the tool moves away from the dome center. In this instance, DRM has the same tool orientation as RM II. This happens when the Darboux frame is rotation-minimizing with respect to the surface normal $\boldsymbol{n}$, and DRM and RM II have the same initial $\beta_{0}$. For the current tool path, it seems that the key to reduce tool orientation changes is to allow the tool inclination angle to vary, which is the case in RM II and DRM but not in RM I. The rate of change of the tool orientation $\left|\boldsymbol{T}^{\prime}\right|$ and the acceleration term $S_{\mathrm{RAB}}$ are plotted in Figs. 16 and 17. In all cases, the values of $\left|\boldsymbol{T}^{\prime}\right|$ and $S_{\mathrm{RAB}}$ are relatively large in the beginning and small toward the end. The curves for RM II and DRM are exactly the same. The peak $\left|\boldsymbol{T}^{\prime}\right|$ and peak $S_{\mathrm{RAB}}$ values for RM I are a few times higher than those for RM II and DRM. They demonstrate that for some surface paths, varying $\alpha$ according to rotation minimization is critical to reducing the tool orientation changes and lightening the burdens on the rotary axes.

\section{Further simulations}

Free-form surfacing is common in five-axis machining. For machining free-form surfaces, the surfaces are typically modelled as spline surfaces. Here, part of the surface is modelled as a bicubic B-spline surface $\boldsymbol{S}(u, v)$ with $0 \leq u, v \leq 1$. The tool path for illustration is generated on the surface using $u(\xi)=v(\xi)=\xi$ with $\xi \in[0,1]$. Tool orientation is rendered along path locations.

The initial tool orientation setup is $\alpha=20^{\circ}$ and $\beta=10^{\circ}$. Besides the three proposed RMF tool orientation methods, Sturz's method (where $\alpha$ is constant, which is also set at $20^{\circ}$, and $\beta=0^{\circ}$ as the tool axis is tilted toward the path tangent) has been adopted in the simulations. Tool orientation along the path is illustrated in Fig. 18 at locations of fixed increments of $\xi$, with the path starting from the left of the surface.

These simulations help elucidate how tool orientation can be adapted using these methods. It seems that RM I and Sturz's method exhibit similar tool orientation changes. This is because $\alpha$ is fixed at the same value for both methods. While $\beta=0^{\circ}$ for Sturz's method, $\beta$ probably varied over a small range for RM I along this particular path. RM II and DRM also seem to have similar tool orientation changes, but they are smaller than those of RM I and Sturz's method, which is confirmed by checking the total tool orientation changes along the path (see Table 1). It is also confirmed that, among the four methods, DRM commanded the least amount of tool orientation changes during the tool path execution. 


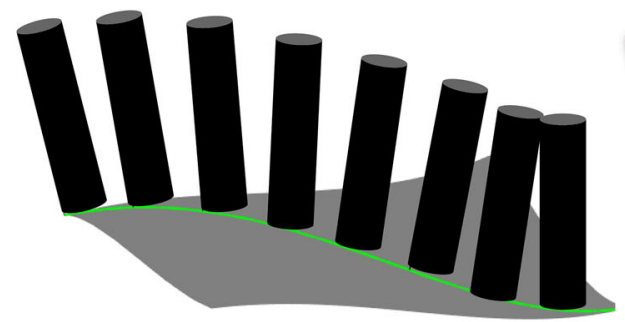

(a)

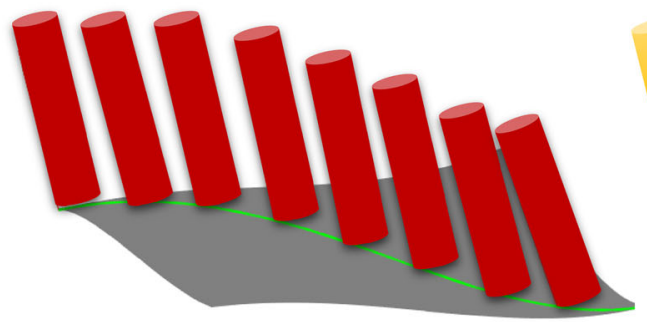

(c)

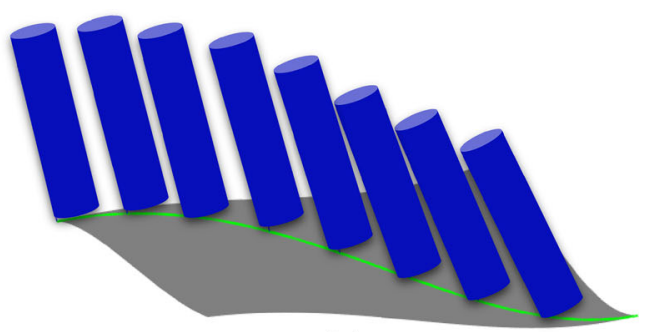

(b)

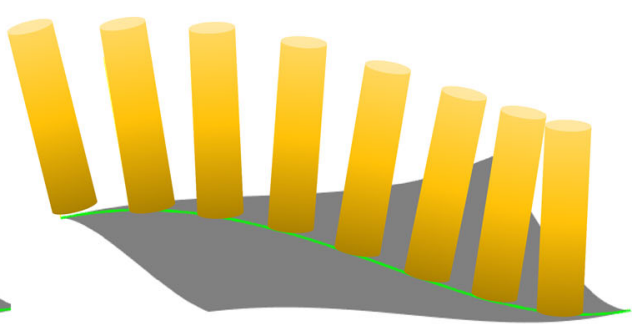

(d)

Fig. 18 Tool orientation along the bicubic B-spline surface path a RM I, b, RM II, c DRM, d Sturz

Table 1 Total tool orientation variation

\begin{tabular}{lllll}
\hline & RM I & RM II & DRM & Sturz \\
\hline Total $\Delta T$ & 140.21 & 61.18 & 28.05 & 142.03 \\
\hline
\end{tabular}

\section{Conclusions}

The concept of RMF was explored for tool orientation planning in the context of five-axis machining. Three RMF methods were presented: RM I (which allows varying the inclination angle), RM II (which allows varying the tilt angle), and DRM (which allows varying both the inclination and tilt angles). These methods specify how tool orientation can be adjusted along the tool path without unnecessary rotation of the tool frame. The derivative of the tool orientation was also derived for these methods.

These methods were applied to flat-end tool machining. Tool orientation was computed for surface paths on a torus, sphere, and dome. Changes in the tool orientation were always smooth for these methods, thus supporting the use of RMF for tool orientation planning. Overall, DRM had the smallest rate of change in tool orientation as well as the smallest acceleration along the rotary axes. The values were sometimes smaller for RM I, and at other times, smaller for RM II. This depends on the local geometry and machine tool kinematics. It is reasonable to expect that to reduce the tool orientation changes and axial acceleration, sometimes, it is more critical to vary the inclination angle, and at other times, the tilt angle. DRM outperforms the other methods because both angles are allowed to vary.
Implementation of these tool orientation methods is rather straightforward as the computation only involves the local geometry of the surface and path. Notwithstanding issues such as collision avoidance, gouging, and cutting quality, which cannot be dealt with in this paper, RMF is an attractive tool for orientation planning. Further, DRM, is particularly useful in five-axis machining. The proposed methods can be extended in a future study to plan stable and smooth tool orientation within the constraints arising from these issues.

Open Access This article is distributed under the terms of the Creative Commons Attribution 4.0 International License (http://crea tivecommons.org/licenses/by/4.0/), which permits unrestricted use, distribution, and reproduction in any medium, provided you give appropriate credit to the original author(s) and the source, provide a link to the Creative Commons license, and indicate if changes were made.

\section{References}

1. Tao C, Ye PQ, Wang JS (2005) Local interference detection and avoidance in five-axis NC machining of sculptured surfaces. Int $\mathbf{J}$ Adv Manuf Technol 25(3-4):343-349

2. Kim YJ, Elber G, Bartoň M et al (2015) Precise gouging-free tool orientations for 5-axis CNC machining. Comput Aided Des 58:220-229

3. Wan M, Liu Y, Xing WJ et al (2018) Singularity avoidance for five-axis machine tools through introducing geometrical constraints. Int J Mach Tools Manuf 127:1-13

4. Liu Y, Wan M, Xing WJ et al (2018) Generalized actual inverse kinematic model for compensating geometric errors in five-axis machine tools. Int J Mech Sci 145:299-317

5. Liu Y, Wan M, Xiao QB et al (2019) Identification and compensation of geometric errors of rotary axes in five-axis machine 
tools through constructing equivalent rotary axis (ERA). Int $\mathbf{J}$ Mech Sci 152:211-227

6. Tunc LT (2019) Smart tool path generation for 5-axis ball-end milling of sculptured surfaces using process models. Robot Comput Integr Manuf 56:212-221

7. Yang P, Yao C, Xie S et al (2016) Effect of tool orientation on surface integrity during ball end milling of titanium alloy TC17. Procedia CIRP 56:143-148

8. Choi BK, Kim DH, Jerard RB (1997) C-space approach to toolpath generation for die and mould machining. Comput Aided Des 29(9):657-669

9. Morishige K, Kase K, Takeuchi Y (1997) Collision-free tool path generation using 2-dimensional $\mathrm{C}$-space for five-axis control machining. Int J Adv Manuf Technol 13(6):393-400

10. Lu J, Cheatham R, Jensen CG et al (2008) A three-dimensional configuration-space method for 5-axis tessellated surface machining. Int J Comput Integr Manuf 21(5):550-568

11. Ezair B, Elber G (2018) Automatic generation of globally assured collision free orientations for 5-axis ball-end tool-paths. Comput Aided Des 102:171-181

12. Chao S, Altintas Y (2016) Chatter free tool orientations in 5-axis ball-end milling. Int J Mach Tools Manuf 106:89-97

13. Lee E (2003) Contour offset approach to spiral tool path generation with constant scallop height. Comput Aided Des 35(6):511-518

14. Li H, Feng HY (1999) Effcient five-axis machining of free-form surfaces with constant scallop height tool paths. Comput Aided Des 31:557-566

15. Ding XM, Fuh JYH, Lee KS (2001) Interference detection for 3-axis mold machining. Comput Aided Des 33(8):561-569

16. Rao A, Sarma R (2000) On local gouging in five-axis sculptured surface machining using flat-end tools. Comput Aided Des 32(7):409-420

17. Lo CC (1999) Effcient cutter-path planning for five-axis surface machining with a flat-end cutter. Comput Aided Des 31(9):557-566

18. Li M, Dong S, Li D (2017) Tool orientation planning method based on divided surface. Mach Des Manuf 174:878-884

19. Castagnetti C, Duc E, Ray P (2008) The domain of admissible orientation concept: a new method for five-axis tool path optimization. Comput Aided Des 40(9):938-950

20. Ho MC, Hwang YR, Hu CH (2003) Five-axis tool orientation smoothing using quaternion interpolation algorithm. Int $\mathrm{J}$ Mach Tools Manuf 43(12):1259-1267

21. Jun CS, Cha K, Lee YS (2003) Optimizing tool orientations for five-axis machining by configuration-space search method. Comput Aided Des 35(6):549-566

22. Hu PC, Tang K (2011) Improving the dynamics of five-axis machining through optimization of workpiece setup and tool orientations. Comput Aided Des 43(12):1693-1706

23. Zhang X, Zhang J, Zheng X et al (2017) Tool orientation optimization of 5-axis ball-end milling based on an accurate cutter/work-piece engagement model. CIRP J Manuf Sci Technol 19:106-116

24. Xu XY (2003) Linear and angular feedrate interpolation for planar implicit curves. Comput Aided Des 35(3):301-317

25. Li LL, Zhang YF, Li HY et al (2011) Generating tool-path with smooth posture change for five-axis sculptured surface machining based on cutter's accessibility map. Int J Adv Manuf Technol 53(5-8):699-709
26. Lin Z, Fu J, Yao X et al (2015) Improving machined surface textures in avoiding five-axis singularities considering tool orientation angle changes. Int J Mach Tools Manuf 98:41-49

27. Farouki RT, Li SQ (2013) Optimal tool orientation control for 5 -axis CNC milling with ball-end cutters. Comput Aided Geom Des 30(2):226-239

28. Farouki RT, Han CY, Li SQ (2014) Inverse kinematics for optimal tool orientation control in 5-axis CNC machining. Comput Aided Geom Des 31(1):13-26

29. Han CY (2016) Tractrix-based tool orientation control for 5-axis CNC machining. Appl Math Comput 272(P1):92-99

30. Farouki RT (2015) Rational rotation-minimizing frames-recent advances and open problems. Appl Math Comput 272(P1):80-91

31. Farouki RT, Giannelli C, Manni C et al (2012) Design of rational rotation-minimizing rigid body motions by Hermite interpolation. Math Comput 81(278):879-903

32. Farouki RT, Giannelli C, Sestini A (2013) An interpolation scheme for designing rational rotation-minimizing camera motions. Adv Comput Math 38(1):63-82

33. Farouki RT, Giannelli C (2009) Spatial camera orientation control by rotation-minimizing directed frames. Comput Anim Virtual Worlds 20:457-472

34. Krajnc M, Vitrih V (2012) Motion design with Euler-Rodrigues frames of quintic Pythagorean-hodograph curves. Math Comput Simul 82(9):1696-1711

35. Jaklic G, Sampoli ML, Sestini A et al (2013) C1 rational interpolation of spherical motions with rational rotation-minimizing directed frames. Comput Aided Geom Des 30(1):159-173

36. Farouki RT, Sakkalis T (2010) Rational rotation-minimizing frames on polynomial space curves of arbitrary degree. J Symb Comput 45(8):844-856

37. Mäurer C, Jüttler B (1999) Rational approximation of rotation minimizing frames using Pythagorean-hodograph cubics. J Geom Graph 3(2):141-159

38. Zheng Z, Wang G (2005) Constructing rotation-minimizing frame of space Bézier curve. J Comput Aid Des Comput Graph 17(8):1785-1792

39. Farouki RT, Sakkalis T (2012) A complete classification of quintic space curves with rational rotation-minimizing frames. J Symb Comput 47(2):214-226

40. Farouki TT, Giannelli C, Sampoli ML (2014) Rotation-minimizing osculating frames. Comput Aided Geom Des 31(1):27-42

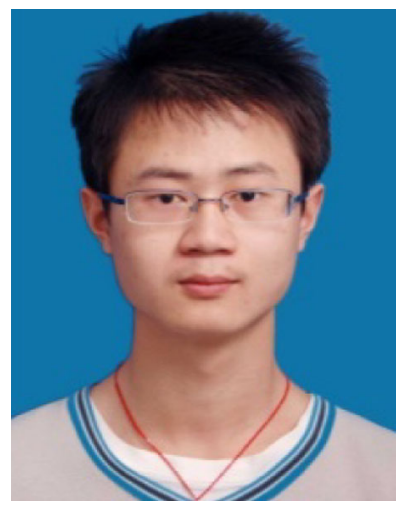

Xiang Zou is a Ph.D. candidate at Institute of Artificial Intelligence and Optimization in School of Computer Science and Technology, Huazhong University of Science and Technology. He obtained his B.S. in Computer Science and Technology from Jiangsu University. His main research interests include optimization, machining learning and fiveaxis CNC machining. 

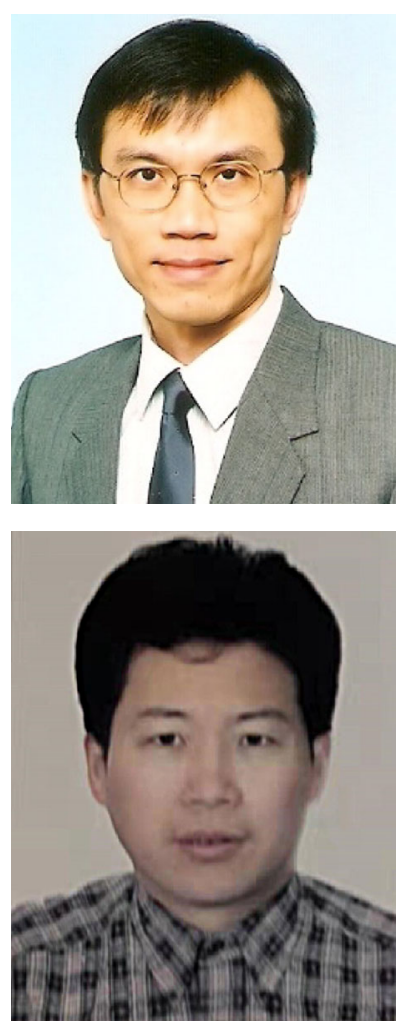

Hon-Yuen Tam received his B.S. degree from Georgia Institute of Technology, USA, and his M.S. and Ph.D. (1990) degrees from Stanford University, USA, all in Mechanical Engineering. He is now a university lecturer of the department of Mechanical Engineering at City University of Hong Kong. His current research interests are in automated surface finishing, numerical control, automation systems and surface inspection.

Hai-Yin Xu obtained his B.Sc. from Northwestern Polytechnical University, M.Sc. and Ph.D. from Huazhong University of Science and Technology PRC. Previously He was a research associate in City University of Hong Kong, a Post doctor in King's College London and a Lecturer in Bournemouth University, UK. He is associate professor at Institute of Artificial Intelligence and Optimization in School of Computer Science and Technology, Huazhong University of Science and Technology. His research interests include optimization, deep neural networks, machining learning and their applications.

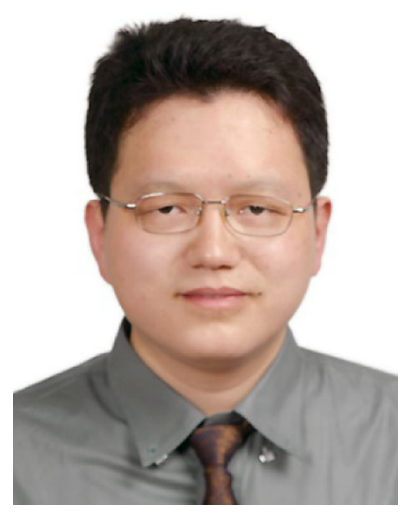

Ke Shi earned his Ph.D. from Huazhong University of Science and Technology and now is a professor of the School of Computer Science and Technology at Huazhong University of Science and Technology. His main research interests include internet of things, big data, and industrial control and automation. 